\title{
A Techno-Economic Analysis of meshed Topologies of Offshore Wind HVAC Transmission.
}

\author{
Stephen Hardy, Dirk Van Hertem, Hakan Ergun \\ KU Leuven Department of Electrical Engineering (ESAT) Heverlee. \\ EnergyVille, Thor Park 8310, 3600 Genk, Belgium
}

\begin{abstract}
This work develops a method of quantifying the effect of adding meshed connections to radial network topologies used for offshore extraction. The method accounts for Capital Expenditures (CAPEX), Corrective maintenance (CM), losses and Expected Energy Not Transmitted (EENT). The stochastic nature of wind is also accounted for. The method is applied to a statistically significant randomly generated population of Offshore Wind Power Plant (OWPP) pairs. Analysis of both Medium Voltage (MV) and High Voltage (HV) bus meshing is performed. It is demonstrated that in the two OWPPs case meshed connections have a narrow but significant region of applicability. Further, the method is applied to a system of 6 wind farms and compared to the lowest cost radial connection, using a number of different planning horizons. It is shown that the optimal radial topology is no longer the clear choice as the development horizon increases.
\end{abstract}

Index Terms-Wind energy, offshore installations, optimization, circuit topology, power transmission.

\section{InTRODUCTION}

Offshore Wind Power Plants (OWPPs) are an essential $\mathrm{CO}_{2}$ reducing technology for meeting the climate targets agreed upon under the The Paris Agreement [1]. In Europe alone, it is estimated that between 240 and $450 \mathrm{GW}$ of offshore wind will be installed by 2050 [2]. Cumulatively to date, only $27 \mathrm{GW}$ have been installed globally with approximately $22 \mathrm{GW}$ of that in Europe [3], [4].

The rate of development has been steadily increasing. In 2019, $3.6 \mathrm{GW}$ of new offshore wind was connected to the European grid, this is the most ever [2]. Outside of Europe, 2.4GW was commissioned in 2019, mostly in China [5]. In February 2019, the world's largest OWPP, the 1.2 GW Horn Sea One became operational [6]. Less than a year later, the construction of the largest collection of OWPPs, namely the $3.6 \mathrm{GW}$ Dogger Bank OWPP cluster in the UK, has begun [7]. Development is accelerating in Asia as well, and the expectation is that China will be the largest offshore wind market before 2030 [4]. In spite of COVID-19, in the first half of 2020, offshore wind development has continued with over $2.5 \mathrm{GW}$ installed globally [4].

For much of the industry's 30 year history, development has focused at the level of individual offshore wind concessions. A government earmarks a region within the national Exclusive

This project has received funding from the European Union's Horizon 2020 research and innovation programme under the Marie Sklodowska-Curie grant agreement no. 765585
Economic Area (EEA) for sea based renewable generation, the region is divided up into concessions and then auctioned off to developers. Developers then attempt to maximize their return for the individual concession. In this model there is little incentive for developers to think in terms of long term offshore grid development. As the industry has matured and the scale of future development becomes clear, however, there is a realization that significant savings and increased reliability are to be gained by a High Voltage (HV) transmission system topology optimized for multiple concessions, considering both current and future developments.

In the UK, movement in this direction was made in 2009 with the unbundling of offshore transmission and generation, creating the new asset class of Offshore Transmission Owner (OFTO). This was done in part to incentivize the development of more coordinated HV grids offshore. Although, a shift towards such grids is desirable, existing research in the field is still in the early stages [8]-[11]. Much work has, however, been done on the related problems of collection circuit topology optimization [12]-[27] and the optimal distribution network planning problem [28]-[32].

Electrical grid topology optimization is a non-linear, nonconvex and mixed-integer problem classified as NP-hard. With such a problem type, it is common to utilize heuristics to provide a high quality solution within an acceptable computation time. Albeit, at the expense of any guarantee on optimality. To this end, Genetic Algorithms (GAs) have been the most frequently applied heuristic [8], [9], [12]-[16], but a wide range of alternative algorithms have also been investigated within the literature, including particle swarm [20], simulated annealing [21], modified bat algorithm [22], modified Clark and Wright's savings algorithm [23], minimal spanning tree [24], branch exchange [30] and path search [31]. In addition to heuristics, classical mathematical formulations have received attention. Much progress has been made using convex relaxations [32][34] and specific formulations using stochastic programming [25], [26], modified vehicular routing Mixed Integer Program (MIP) [27] and cascading, sequential MIP [10] have been implemented among others.

Existing optimization models suffer from either a lack of global optimality or optimality within an overly restricted search space. Furthermore, a focus on singular optimal solutions in isolation is less informative for decision makers than a set of feasible solutions. As such, in this work, an expansion of 


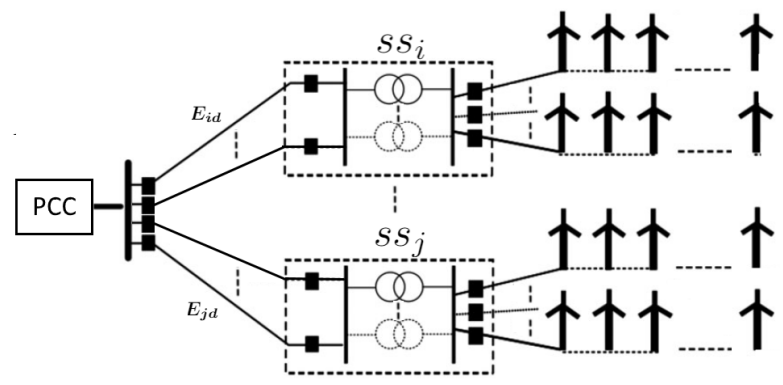

Fig. 1. Adjusted Power Injection Profile In Meshed Topology.

the radial optimization method described in [11] is developed to allow the inclusion of meshed connections. The algorithm in [11] can guarantee global optimality for a single transmission voltage and returns a hierarchy of radial topological options rather than a singular solution. As the optimal radial solution is known, the relative quality of meshed topologies can therefore also be quantified. As the economic model implemented is identical to that of the base algorithm readers are referred to the original paper for details.

The developed method is investigated using a statistical analysis to access the performance of meshed configurations on pairs of OWPPs, where parameters such as power rating and distance from shore are varied. A statistically relevant set of variations is ensured using the bootstrap sampling method allowing the economically viable regions for adopting meshed connections to be determined. This is further validated using a test case with six OWPPs showing that meshed OWPP connections can provide economic benefits.

The remainder of this paper is structured as follows. In section II the modelling methodology is described. This is followed by a description of the sampling process employed to obtain a large set of randomly generated pairs of OWPPs used for the statistical analysis. The results are presented in section IV. First, those from the statistical analysis and then those for three resulting topologies of a region of six OWPPs. As it is not realistic to develop such a region within a single time step, development over six and ten year horizons are analyzed. Finally, conclusions of the work are highlighted.

\section{MOdel Description}

\section{A. Reliability of Meshed Topologies}

A radial topology consisting of two or more OWPPs connected to a point of common coupling (PCC) is shown in fig 1 . Meshed connections can be introduced between any two nonserial connected Offshore Substations (OSSs): $s s_{i}$ and $s s_{j}$. The set of equipment, $\boldsymbol{E}_{\boldsymbol{i d}}$, making up the radial connection between $s s_{i}$ and d, the demand at the PCC is shown in fig 1 and described by:

$$
\boldsymbol{E}_{\boldsymbol{i d}}=\left\{e_{i i-1}, e_{i-1 i-2}, \ldots, e_{1 d}\right\}
$$

The size and cost of equipment in $\boldsymbol{E}_{\boldsymbol{i d}}$ is calculated according to the economic model presented in [11]. Additionally, two sets, $\overline{\boldsymbol{E}_{\boldsymbol{i d}}}$ and $\boldsymbol{E}_{\boldsymbol{j} \boldsymbol{d}}$ are defined. $\overline{\boldsymbol{E}_{\boldsymbol{i d}}}$ is the set of equipment, one size increment larger from those in $\boldsymbol{E}_{\boldsymbol{i d}}$, e.g. a $400 \mathrm{~mm}^{2}$ cable is replaced by a $500 \mathrm{~mm}^{2}$ cable. $\boldsymbol{E}_{\boldsymbol{i d}}$ is the set of equipment, one size increment smaller. The set of candidate topologies is then defined by matrix (2).

$$
T_{i j}=\left[\begin{array}{ccc}
E_{i d}, E_{j d} & \overline{E_{i d}}, E_{j d} & \underline{E_{i d}}, \underline{E_{j d}} \\
E_{i d}, \overline{E_{j d}} & \overline{E_{i d}}, \underline{E_{j d}} & \underline{E_{i d}}, \underline{E_{j d}} \\
E_{i d}, \underline{E_{j d}} & \overline{E_{i d}}, \underline{E_{j d}} & \underline{E_{i d}}, \underline{E_{j d}}
\end{array}\right]
$$

The rating of a meshed connection, $S_{i j}^{\#}$, is given by (3), where $S_{i d}$ and $S_{i}^{g}$ are the transmission capacity of the radial connection to the PCC from $s s_{i}$ and the sum of the peak injected power at $s s_{i}$, respectively.

$$
S_{i j}^{\#}=\max \left(\left|S_{i d}-S_{i}^{g}\right|,\left|S_{j d}-S_{j}^{g}\right|\right)
$$

Equation (3) ensures that the meshing cable is of sufficient size to transmit the expected peak injection in case of undersized radial connectors, or to provide sufficient capacity to utilize excess capacity in case of oversized radial connectors. The first condition that must be met by a candidate meshed topology is that the cost of the extension forming the mesh be less than the cost of the Expected Energy Not Transmitted (EENT) of the radial connections being meshed. This is expressed by:

$$
f\left(c b_{i j}^{t t l}\right)-f\left(c b_{i j}^{e e n t}\right) \leq \sum_{e_{i i-1} \in E_{i d}} f\left(e_{i i-1}^{e e n t}\right)+\sum_{e_{j j-1} \in E_{j d}} f\left(e_{j j-1}^{e e n t}\right)
$$

Where $f(x)$ is the cost of $\mathrm{x}$ and $c b_{i j}$ is the meshing cable from $s s_{i}$ to $s s_{j}$. For candidate meshing cables satisfying condition (4) the reduction in EENT is calculated as follows. Considering each possible contingency, $c$, i.e. failure of a cable or transformer, at five evenly spaced OWPP generation levels, $g$, from 20 to $100 \%$, a yearly power flow profile similar to fig. 2 is determined by solving the linearized Optimal Power Flow (OPF) problem for power delivered to the PCC. As the OWPPs are geographically proximate the wind speeds are considered highly correlated and therefore the generation levels are varied in unison. To ensure maximum power transfer, demand at the PCC is maximized, (5), subject to transmission constraints (6).

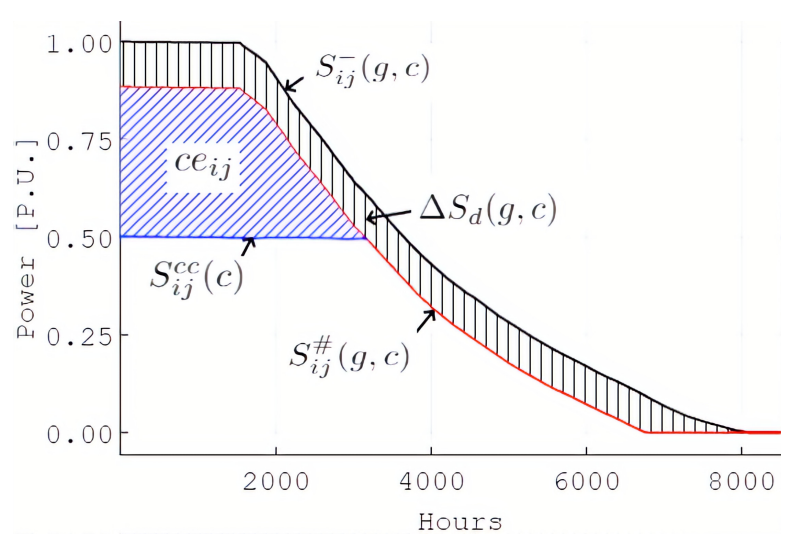

Fig. 2. Adjusted power injection profile in meshed topology. 


$$
\left.\begin{array}{cc}
\max S_{d} & \\
S_{i}^{g}-S_{i}^{d}=\sum_{(i, j) \in E} S_{i, j}-\sum_{(j, i) \in E} S_{j, i} & \\
S_{i j}=\sum_{(j, i) \in E} B_{i j}\left(\theta_{i}-\theta_{j}\right) & \\
S_{i}^{g, m i n} \leq S_{i}^{g} \leq S_{i}^{g, \max } & \forall i \in N \\
S_{i j}^{\text {min }} \leq S_{i j} \leq S_{i j}^{\max } & \forall g \in G \\
V_{i}^{\text {min }} \leq V_{i} \leq V_{i}^{\max } & \forall(i, j) \in E \\
\theta_{i}^{\text {min }} \leq \Delta \theta_{i} \leq \theta_{i}^{\max } & \forall \\
\end{array}\right\}
$$

For a contingency $c$ and a generation level $g$, the difference in power delivered to the PCC in the radial and meshed cases, $\Delta S_{d}(g, c)$, is the additional transmission capacity provided by the meshed connection. This amount is subtracted from the power injected into equipment $\mathrm{e}_{i j}^{-}$, in the radial connection to obtain the power injected into $\mathrm{e}_{i j}^{\#}$ in the meshed connection as in:

$$
\begin{aligned}
& S_{i j}^{\#}(g, c)=S_{i j}^{-}(g, c)-\Delta S_{d}(g, c) \\
& \text { where } \Delta S_{d}(g, c)=S_{d}^{\#}(g, c)-S_{d}^{-}(g, c) .
\end{aligned}
$$

Constrained energy $(c e)$ is then obtained by taking the sum of the difference between $S_{i j}^{\#}(g, c)$ and the constrained capacity $S_{i j}^{c c}(c)$, for all hours within a year in which generation, $g_{h}$, exceeds the constrained capacity as in:

$$
\begin{gathered}
c e_{i j}=\sum_{h \in H}\left(S_{i j}^{\#}\left(g_{h}, c\right)-S_{i j}^{c c}(c)\right) \\
H=\left\{h \in\{1,2,3, \ldots, 8760\} \mid S_{i j}^{\#}\left(g_{h}, c\right)>S_{i j}^{c c}(c)\right\}
\end{gathered}
$$

The EENT within the meshed topology is then calculated as in (9). Where $\pi_{i j}$ is the probability of a given contingency state and $k_{e p}$ is the cost of energy, assumed the same in this work as

\begin{tabular}{|c|c|c|c|c|c|}
\hline$C$ & $S_{i j}^{c c}$ & $\pi_{i j}$ & Equipment & $\lambda[1 / y r]$ & $\mu[$ months $]$ \\
\hline 1 & $\mathrm{~S}$ & $\Lambda_{i j}$ & Transformer & 0.02 & PCC: 0.6 , OSS: 3 \\
\hline 0 & 0 & $1-\Lambda_{i j}$ & Cable & $0.001 / \mathrm{km}$ & 2 \\
\hline
\end{tabular}
in [35], to be $50 € / \mathrm{MWh}$. To simplify the calculation of $\pi_{i j}$ it is convenient to construct a Capacity Outage Probability Table (COPT) similar to that in table I. As contingencies up the degree N-N are considered, the number of contingency states, $C$, for a piece of equipment, $\mathrm{e}_{i j}$, is equivalent to $2^{n}$. Where $\mathrm{n}$ is the number of parallel circuits in $\mathrm{e}_{i j}$. The probability of each state is obtained via (10) and table II, where $\lambda_{i j}$ and $\mu_{i j}$ are the failure rate and the mean time to repair respectively.

$$
\begin{gathered}
E E N T_{i j}=8760 k_{e p} \sum_{(i, j) \in E} c e_{i j} \pi_{i j} . \\
\Lambda_{i j}=\frac{1}{1+\lambda_{i j} \cdot \frac{\mu_{i j} \cdot 30 \cdot 24}{8760}} \mid \forall(i j) \in E,
\end{gathered}
$$

TABLE I EXAMPLE COPT

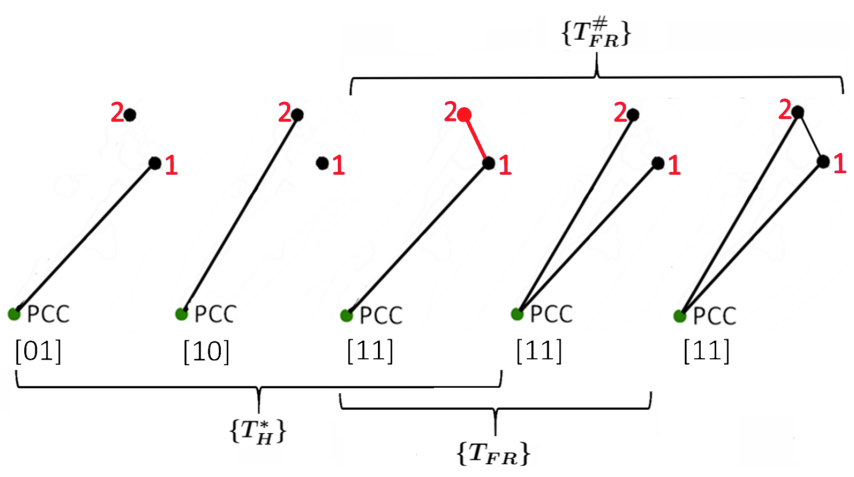

Fig. 3. Representative sets $\left\{\boldsymbol{T}_{\boldsymbol{H}}^{*}\right\},\left\{\boldsymbol{T}_{\boldsymbol{F} R}\right\}$ and $\left\{\boldsymbol{T}_{\boldsymbol{F} R}^{\#}\right\}$ for 3 OWPPs.

\section{B. Greedy Search with Meshed Connections}

The greedy search algorithm described in [11] finds the set $\left\{T_{H}^{*}\right\}$, which contains all optimal radial topologies for all combinations of $i \leq n$ OWPPs, utilizing a single connection to the PCC. This is visualized for the two OWPPs case in fig. 3 . The full rank ( $i=n$ OWPPs) optimal radial connection, $t^{*}$, utilizing $i \leq n$ connections to the PCC is found as in (11).

$$
\begin{aligned}
& t^{*}=\min (f(t)), \forall t \in\left\{\boldsymbol{T}_{\boldsymbol{F} \boldsymbol{R}}\right\}, \text { where } \\
& \left\{\boldsymbol{T}_{\boldsymbol{F R}}\right\}=\left\{t=\left(\begin{array}{c}
\left\{\boldsymbol{T}_{k}^{*}\right\} \\
k
\end{array}\right) \mid \sum_{\in k} j=2^{n}-1\right\} .
\end{aligned}
$$

Here, $j$ is a binary number where the number of the OWPP corresponds to the position within the binary number. OWPPs are ordered from nearest to farthest from the PCC. If a particular OWPP is connected within a topology, its corresponding position in $j$ is 1 , else it is 0 . The values of $j$ for the two OWPPs case are shown below each topology in fig. 3. $\left\{T_{F R}\right\}$ is the set of all full rank radial topologies of $i \leq n$ connections to the PCC. When constructing $\left\{\boldsymbol{T}_{\boldsymbol{F R}}\right\}$, topologies with greater than one connection to the PCC are analyzed for meshed connections between all non-serially connected OSSs using the previously described method. The set $\left\{T_{F R}^{\#}\right\}$, is then the union between $\left\{T_{F R}\right\}$ and any additional meshed topologies. Refer again to fig. 3 for the visual intuition of $\left\{T_{F R}^{\#}\right\}$.

\section{Population SAMPling}

Two sets of random populations of topologies, for $220 \mathrm{kV}$ and $400 \mathrm{kV}$ levels are generated and analyzed for Medium Voltage (MV) and HV bus meshing. The offshore regions investigated consist of two OWPPs connected to a single PCC. The exhaustive search space, $\left\{T^{e x}\right\}$, from which the populations are sampled is described by (12) with input variables visualized in fig. 4 . 


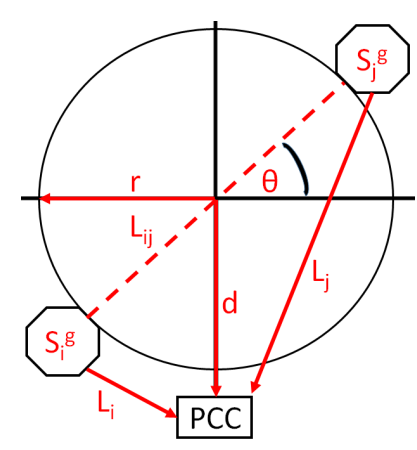

Fig. 4. Meshed topology domain.

$$
\begin{gathered}
\left\{\boldsymbol{T}^{e x}\right\}=f\left(S_{i}^{g}, S_{j}^{g}, r, d, \theta\right) \text { where: } \\
S_{i}^{g} \text { and } S_{j}^{g} \in\{200,210, \ldots 990,1000\} \text { [MVA] } \\
0.5 \leq r \leq 15 \mathrm{~km}, 20 \leq d \leq 100 \mathrm{~km},-\pi \leq \theta \leq \pi
\end{gathered}
$$

A sample offshore region is obtained by randomly generating values for the OWPPs capacities: $S_{i}^{g}, S_{j}^{g}$ and their distances from shore and each other. Both of which are functions of $r$, $d$ and $\theta$. As a sampling method, sampling with replacement as in [36] is used. A total of 16513 samples at each voltage level is selected in a $(1-\alpha)$ confidence interval of $99 \%$, meaning that the sampling error becomes less than $1 \%$ according to (13), where $z_{\alpha / 2}$ is the Z-statistic.

$$
N \geq \frac{z_{\alpha / 2}^{2}}{4 e^{2}}
$$

For each sample offshore region, the three topologies comprising the set $\left\{T_{F R}^{\#}\right\}$ in fig. 3 are calculated. Moving from left to right in the figure these topologies are referred to as: radial connection 2 (RT2), radial connection 1 (RT1) and the meshed connection (MT1).

\section{NumericAl Results}

\section{A) Statistical Analysis of the Random Population}

As MT1 is derived from RT1, it is most insightful to first compare these two variations in isolation and identify when the addition of a meshed connection results in a lower total system cost. The following results are therefore only considering RT1 and MT1. At the end of this section, the results including RT2 will be discussed.

The composition of the lowest cost topologies within the studied population can be seen in table III. RT1 connections include ones with two fully sized export cables and those with at least one that is undersized. Only MT1 connections that result in greater than a $1 \%$ decrease in overall cost are considered desirable.

In table IV the export cable sizing of desirable meshed topologies is shown. In all cases a meshed connection is only cost effective if one of the export cables is undersized. Furthermore, in the vast majority of these topologies it is the higher capacity export cable that is undersized (as $S_{i}^{g}$ is always
TABLE III

PERCENT COMPOSITION OF RANDOM POPULATION WHERE MT1 OR RT1 ARE THE LOWEST COST TOPOLOGY.

\begin{tabular}{lll|ll} 
& $\mathbf{2 2 0} \boldsymbol{k} \boldsymbol{V}$ & & $\mathbf{4 0 0} \boldsymbol{k} \boldsymbol{V}$ & \\
& MT1 & RT1 & MT1 & RT1 \\
\hline MV & 1.71 & 70.71 & 2.51 & 75.19 \\
HV & 2.51 & 25.07 & 7.09 & 15.21 \\
\hline Total & 4.22 & 95.78 & 9.61 & 90.40
\end{tabular}

\begin{tabular}{|c|c|c|c|c|c|c|}
\hline $\begin{array}{c}\text { Export } \\
{[\mathrm{kV}]}\end{array}$ & $\begin{array}{c}\text { Mesh } \\
{[\mathrm{kV}]}\end{array}$ & $\frac{E_{i d}}{E_{j d}}$, & $\begin{array}{l}E_{i d} \\
E_{j d}\end{array}$ & $\frac{E_{i d}}{E_{j d}}$ & $\begin{array}{l}\overline{E_{i d}}, \\
E_{j d}\end{array}$ & $\frac{E_{i d}}{\overline{E_{j d}}}$ \\
\hline 220.0 & 66.0 & $\overline{0.35}$ & $\overline{92.93}$ & 6.71 & $\overline{0.7}$ & 0.0 \\
\hline 220.0 & 220.0 & 0.0 & 72.29 & 26.7 & 0.48 & 0.48 \\
\hline 400.0 & 66.0 & 1.21 & 71.3 & 24.8 & 1.67 & 0.96 \\
\hline 400.0 & 400.0 & 0.0 & 68.92 & 31.08 & 0.0 & 0.0 \\
\hline
\end{tabular}

TABLE IV

EXPORT CABLE CONFIGURATION FOR MV AND HV MESHED CONNECTIONS AS PERCENTAGE OF RESPECTIVE POPULATIONS.

less than or equal in capacity to $S_{j}^{g}$ ). A negligible number of topologies benefit from the alternate cable having an increased capacity or both export cables being undersized.

At $220 \mathrm{kV}$, it is rare that the addition of a meshed connection results in a lower cost topology, occurring in only $4.22 \%$ of cases. Among these cases, HV bus meshing is most frequently the better option. This despite MV bus meshing providing increased reliability for transformers as well as the HV export cables compared to just the export cables with an HV bus mesh. This is due to the high cost per watt of MV cable.

The average savings at $220 \mathrm{kV}$ from an accepted MT1 connection is $2.6 \%$ with a median of $2.1 \%$. The maximum savings found was $14.7 \%$. This occurred at $S_{i}^{g}=340 \mathrm{MVA}$, $S_{j}^{g}=370 \mathrm{MVA}, r=1.5 \mathrm{~km}, d=93 \mathrm{~km}$ and $\theta=-50^{\circ}$.

Although the benefit of meshing at $220 \mathrm{kV}$ transmission is limited, the cases where meshing is warranted falls within a highly applicable offshore region. The most desirable meshed connections, those resulting in savings of at least $10 \%$, all have capacities between $340 \mathrm{MVA}$ and 390 MVA located between 40 and $100 \mathrm{~km}$ offshore. Globally, the average rating of OWPPs under construction is 370 MVA [4]. In fig. 5 (B) this range for an OSS to OSS distance of $1 \mathrm{~km}$ is shown, while in (A) the dependence on the OSS to OSS distance for $S_{i}^{g}=S_{j}^{g}=360$ MVA is demonstrated.

Closer investigation of this region, reveals that it is the finite step sizes of cables that cause MT1 to be the lower cost topology in this range. Meshing becomes effective at the transition between a single $1000 \mathrm{~mm}^{2}$ 3-core cable and two parallel $400 \mathrm{~mm}^{2} 3$-core cables. At this transition point, the export cable has a very low loading ratio approaching $70 \%$. The distance at which this transition occurs for various capacities can be seen clearly in fig. 5 (B), where there is an abrupt change from black to yellow or orange.

At $400 \mathrm{kV}$ transmission, beneficial meshed connections are more frequent, occurring in $9.61 \%$ of cases. This is due to limited available cable sizes compared to $220 \mathrm{kV}$. The average savings among these connections is $6.5 \%$ with a median of $5.3 \%$. The maximum savings found was $21.1 \%$. This occurred at $S_{i}^{g}=220 \mathrm{MVA}, S_{j}^{g}=570 \mathrm{MVA}, r=0.5 \mathrm{~km}, d=96 \mathrm{~km}$ and 


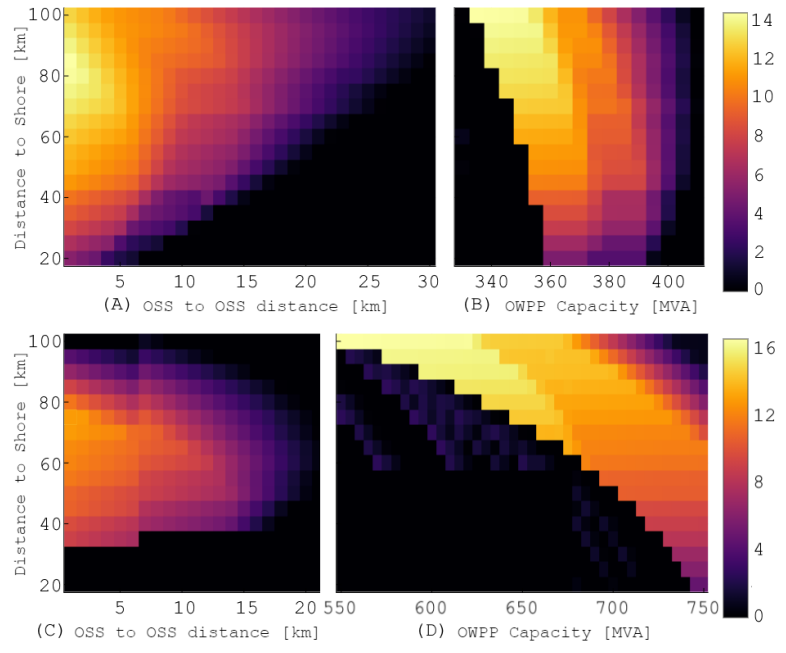

Fig. 5. Percent savings distribution of MT1 over RT1 at $\theta=0$. Top: $220 \mathrm{kV}$. Bottom: $400 \mathrm{kV}$. (A) $-S_{i}^{g}=S_{j}^{g}=360 \mathrm{MVA}$. (C) $-S_{i}^{g}=S_{j}^{g}=720 \mathrm{MVA}$. (B), (D) - The $s s_{i}$ to $s s_{j}$ distance is $1 \mathrm{~km}$.

$\theta=-45^{\circ}$. Despite MT1 being the lowest cost option in a larger percentage of the search space at $400 \mathrm{kV}$, the range of capacities that benefit most are in a less practical region compared to $220 \mathrm{kV}$. The connections most highly benefited, resulting in savings of $15 \%$ or more consist almost entirely of low capacity OWPPs, less than 340 MVA, paired with high capacity ones, greater than $530 \mathrm{MVA}$. As the lowest available cable size at $400 \mathrm{kV}$ included in this study is single core $500 \mathrm{~mm}^{2}$, which has a capacity at $50 \mathrm{~km}$ of over $500 \mathrm{MVA}$ this is not a surprising result. The loading ratio of $400 \mathrm{kV}$ export cables connecting OWPPs between 200 and 340 MVA is at best just slightly over $70 \%$ and at worst less than $40 \%$. A more applicable region at $400 \mathrm{kV}$ transmission occurs when both OWPPs are greater than 500 MVA. This region is displayed in the lower half of fig. 5 and accounts for $40 \%$ of all selected MT1 with a savings greater than $10 \%$. The transition visible in (D) is the point where a single $2000 \mathrm{~mm}^{2}$ cable is no longer of sufficient capacity. (E) shows the impact of OSS to OSS distance at $S_{i}^{g}=S_{j}^{g}=720 \mathrm{MVA}$.

When RT2 is included in the comparison MT1 is only the lowest cost option $0.56 \%$ of the time at $220 \mathrm{kV}$ and $1.39 \%$ of the time at $400 \mathrm{kV}$. RT2 was the lowest cost option in $38 \%$ of $220 \mathrm{kV}$ cases and in $51 \%$ of $400 \mathrm{kV}$ configurations. An important observation is that the median distance between OWPPs where MT1 was the globally optimal topology increased from $14 \mathrm{~km}$ to $20 \mathrm{~km}$ for $220 \mathrm{kV}$ and $12 \mathrm{~km}$ to $19 \mathrm{~km}$ for $400 \mathrm{kV}$. This is because for closely situated OWPPs, the lowest cost option is to extend the MV collection grid to a single centrally located OSS.

\section{B) Topology with six OWPPs}

To investigate the algorithm on offshore regions of greater than two OWPPs, a region consisting of 6 OWPPs is simulated. A capacity of 360 MVA for each OWPP was selected as it is both near the average capacity of OWPPs being installed

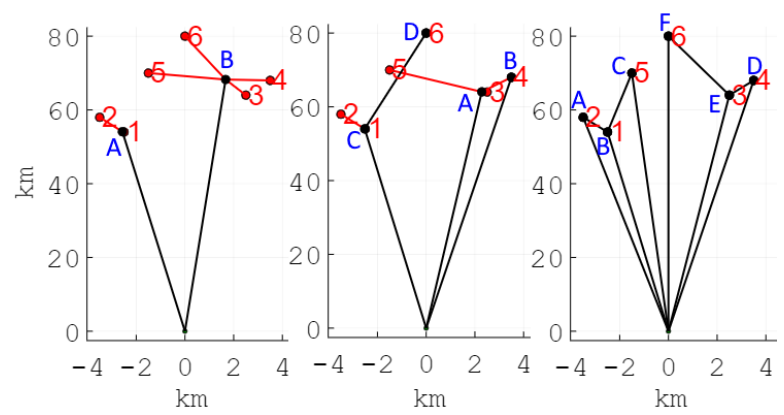

Fig. 6. RT1 (left), MT1 (centre), MT2 (right).

world wide and because within the studied population, meshed topologies at this capacity had a wide range where they tented to perform well, see the top of fig. 5. The transmission voltage is $220 \mathrm{kV}$ and the collection grid voltage is $66 \mathrm{kV}$. The resulting set of feasible topologies, $\left\{T_{F R}^{\#}\right\}$, consists of 863 topologies. Three topologies among this hierarchy are presented forthwith; the lowest cost radial topology (RT1), the lowest cost topology with at least one meshed connection (MT1) and the topology with the highest number of meshed connections (MT2). These topologies are shown in fig. 6. The OWPPs are labelled 1 to 6 and any OSS with letters A through F. HV export cables are shown in black and MV export cables in red.

A cost breakdown of the three topologies is presented in table V. RT1 has the overall lowest cost due to lower Capital Expenditures (CAPEX) and Corrective Maintenance (CM). The lowest overall EENT and losses are found within MT1, but by only a small margin. It is likely that RT1 is the globally optimal topology from a purely cost perspective, however, RT1 has the disadvantage that large upfront CAPEX is required, particularly for the two OSSs. As such, an analysis of two different time horizons is performed, namely six and ten years. The OWPPs are assumed to be commissioned in six equally spaced time steps. One OWPP per time step. Over the six year development period, one OWPP is commissioned per year and in the ten year development period, one OWPP is commissioned approximately every twenty months. When export cables consist of parallel circuits, the minimum number of cables is installed per time step to sufficiently service all currently commissioned OWPPs. If this were not done the radial connection would be at a significant disadvantage. The discount rates used of $6 \%$ and $9.5 \%$ are the minimum and maximum rates for offshore wind as reported in [37]. The results of the analysis are shown in table VI. Over a six year development horizon the radial topology is still the best choice. For a ten year development plan with a discount rate approaching $9.5 \%$, MT1 becomes the better choice.

\section{Conclusions}

In this work a method to quantify the effect of meshed connections on radial offshore wind transmission topologies in terms of a reduction in EENT has been presented. The method is applied to a large randomly generated population of OWPP pairs and the results were analyzed statistically. 
TABLE V

COST BREAKDOWN FOR RADIAL AND MESHED TOPOLOGIES CONSIDERING SINGLE INVESTMENT.

\begin{tabular}{llll} 
& RT1 & MT1 & MT2 \\
\hline CAPEX & 1173.3 & 1186.1 & 1244.08 \\
LOSSES & 56.34 & 55.6 & 59.30 \\
C.M. & 219.78 & 226.7 & 237.65 \\
EENT & 56.33 & 54.65 & 64.46 \\
\hline Total: & 1506.02 & 1523.1 & 1605.49
\end{tabular}

TABLE VI

Net Present Value of multi year Offshore Development.

\begin{tabular}{ccccc}
$\begin{array}{c}\text { Discount } \\
\text { Rate }[\%[\end{array}$ & $\begin{array}{c}\text { Development } \\
\text { Period [years }]\end{array}$ & $\begin{array}{c}\text { RT } \\
{[\mathrm{M} €[}\end{array}$ & $\begin{array}{c}\text { MT1 } \\
{[\mathrm{M} €[}\end{array}$ & $\begin{array}{c}\text { MT2 } \\
{[\mathrm{M} €[}\end{array}$ \\
\hline 6 & 6 & 1247.28 & 1325.64 & 1382.12 \\
9.5 & 6 & 1190.03 & 1232.33 & 1283.23 \\
6 & 10 & 1179.39 & 1215.3 & 1265.17 \\
9.5 & 10 & 1095.68 & 1084.73 & 1126.71 \\
\hline
\end{tabular}

It was shown that the region where the meshed topology is the optimal topology is small, particularly at $220 \mathrm{kV}$, but it is still relevant as it is at capacities centered around the global average OWPP capacity. Meshed connections were found to only be beneficial if at least one of the export cables being meshed was undersized. In addition, HV bus meshing was shown to be preferable to MV bus meshing, particularly as at close distances the lowest cost topology is generally a single larger OSS with MV cable feeding it directly from both OWPPs. The method was further validated on a region of six OWPPs. Under the premise that all investments are being conducted at the same time, the radial topology is cheapest option. Nevertheless, if sequentiality of investments over a number of years is considered, meshed topologies become economically more viable.

\section{REFERENCES}

[1] UNCCC, "The Paris Agreement," uNTC XXVII 7.d.

[2] Walsh et al., "Offshore Wind in Europe, key trends and statistics 2019," Wind Europe, Tech. Rep., 2020.

[3] Komusanac, Brindley, Fraile, "Wind Energy in Europe in 2019, trends and statistics," Wind Europe, Tech. Rep., 2020.

[4] G. Herzig., "Global offshore wind report 1st half 2020." World Forum Offshore Wind., Tech. Rep., 2020.

[5] "Global offshore wind report 2020," Global Wind Energy Council, Tech. Rep., 2020.

[6] "Hornsea project one, north sea," https://www.powertechnology.com/projects, accessed: 20-04-06.

[7] "Dogger bank wind farm," https://doggerbank.com/, accessed: 20-04-03.

[8] Lingling, Yang, Xiaoming, "Optimization of electrical connection scheme for large offshore wind farm with genetic algorithm." IEEE, 2009, pp. 1-4.

[9] H. Ergun, D. Van Hertem, and R. Belmans, "Transmission system topology optimization for large-scale offshore wind integration," IEEE Transactions on Sustainable Energy, vol. 3, no. 4, pp. 908-917, 2012.

[10] Hardy, Ergun, Van Hertem, "A Techno-Economic MILP Optimization of Multiple Offshore Wind Concessiosn." Large-Scale Grid Integration of Renewable Energy in India, 2019.

[11] _ , "A greedy algorithm for calculating an optimallybounded from below hierarchy of transmissionnetwork topologies for offshore wind power." IEEE Transactions on Power Systems 2020 (Under review), 2020.

[12] C. Szafron, "Offshore windfarm layout optimization." IEEE, 2010, pp. $542-545$.

[13] J. Gonzalez, A. Rodriguez, J. Mora, J. Santos, and M. Payan, "A new tool for wind farm optimal design." IEEE, 2009, pp. 1-7.

[14] C. Elkinton, J. Manwell, and J. Mcgowan, "Optimizing the layout of offshore wind energy systems," Marine Technology Society Journal, vol. 42, pp. 19-27, 062008.
[15] O. Dahmani, S. Bourguet, M. Machmoum, P. Guerin, P. Rhein, and L. Josse, "Optimization of the connection topology of an offshore wind farm network," IEEE Systems Journal, vol. 9, no. 4, pp. 1519-1528, 2015.

[16] _ "Optimization and reliability evaluation of an offshore wind farm architecture," IEEE Transactions on Sustainable Energy, vol. 8, no. 2, pp. 542-550, 2017.

[17] A. Masmoudi, "2014 ninth international conference on ecological vehicles and renewable energies foreword," IEEE Transactions on Magnetics, vol. 51, no. 4, pp. 1-1, 2015.

[18] S. Lumbreras and A. Ramos, "Offshore wind farm electrical design: a review: Offshore wind farm electrical design," Wind energy (Chichester, England), vol. 16, no. 3, pp. 459-473, 2013.

[19] Hopewell et. al, "Optimising the design of offshore wind farm collection networks," vol. 1. IEEE, 2006, pp. 84-88.

[20] X. Gong, S. Kuenzel, and B. C. Pal, "Optimal wind farm cabling," IEEE transactions on sustainable energy, vol. 9, no. 3, pp. 1126-1136, 2018.

[21] Yang and Cho, "Simulated annealing algorithm for wind farm layout optimization: A benchmark study," Energies (Basel), vol. 12, no. 23, p. 4403, 2019.

[22] Z. Chen, C. Chen, W. Hu, and P. Hou, "Optimisation of offshore wind farm cable connection layout considering levelised production cost using dynamic minimum spanning tree algorithm," IET renewable power generation, vol. 10, no. 2, pp. 175-183, 2016.

[23] T. Zuo, Y. Zhang, K. Meng, and Z. Y. Dong, "Collector system topology for large-scale offshore wind farms considering cross-substation incorporation," IEEE transactions on sustainable energy, vol. 11, no. 3, pp. $1601-1611,2020$

[24] Y. Qi, P. Hou, L. Yang, and G. Yang, "Simultaneous optimisation of cable connection schemes and capacity for offshore wind farms via a modified bat algorithm," Applied sciences, vol. 9, no. 2, p. 265, 2019.

[25] M. Banzo and A. Ramos, "Stochastic optimization model for electric power system planning of offshore wind farms," IEEE Transactions on Power Systems, vol. 26, no. 3, pp. 1338-1348, 2011.

[26] Lumbreras et. al, "A benders' decomposition approach for optimizing the electric system of offshore wind farms." IEEE, 2011, pp. 1-8.

[27] J. Bauer and J. Lysgaard, "The offshore wind farm array cable layout problem: a planar open vehicle routing problem," The Journal of the Operational Research Society, vol. 66, no. 3, pp. 360-368, 2017.

[28] Bahadoorsingh et al., "Minimization of voltage sag costs by optimal reconfiguration of distribution network using genetic algorithms," IEEE Transactions on Power Delivery, vol. 22, no. 4, pp. 2271-2278, 2007.

[29] D. Jakus, R. Čaenović, J. Vasilj, and P. Sarajčev, "Optimal reconfiguration of distribution networks using hybrid heuristic-genetic algorithm," Energies (Basel), vol. 13, no. 7, p. 1544, 2020.

[30] Edelmiro et al., "An improved branch-exchange algorithm for large-scale distribution network planning," IEEE transactions on power systems, 2002.

[31] Vikrant et al., "Optimization of radial distribution networks using path search algorithm," International Journal of Electronics and Electrical Engineering, 2013.

[32] J. A. Taylor and F. S. Hover, "Convex models of distribution system reconfiguration," IEEE Transactions on Power Systems, vol. 27, no. 3, pp. 1407-1413, 2012.

[33] H. Ergun, J. Dave, D. Van Hertem, and F. Geth, "Optimal power flow for ac-dc grids: Formulation, convex relaxation, linear approximation, and implementation," IEEE transactions on power systems, vol. 34, no. 4, pp. 2980-2990, 2019.

[34] J. Dave, H. Ergun, and D. Van Hertem, "Relaxations and approximations of hvdc grid tnep problem." PSCC, 2020.

[35] Flament et al., "North sea grid, final report." 3E, Tech. Rep., 2014.

[36] Y. Li and R. P. Gopalan, "Effective sampling for mining association rules," in AI 2004: Advances in Artificial Intelligence, ser. Lecture Notes in Computer Science. Berlin, Heidelberg: Springer Berlin Heidelberg, 2005, vol. 3339, pp. 391-401.

[37] "Renewable energy discount rate survey results," Grant Thornton, Tech. Rep., 2018. 